\title{
Light Propagation in Inhomogeneous Universes. V. Gravitational Lensing of Distant Supernovae.
}

\author{
Hugo Martel ${ }^{1}$ and Premana Premadi ${ }^{2}$
}

\begin{abstract}
We use a series of ray-tracing experiments to determine the magnification distribution of high-redshift sources by gravitational lensing. We determine empirically the relation between magnification and redshift, for various cosmological models. We then use this relation to estimate the effect of lensing on the determination of the cosmological parameters from observations of high- $z$ supernovae. We found that, for supernovae at redshifts $z<1.8$, the effect of lensing is negligible compared to the intrinsic uncertainty in the measurements. Using mock data in the range $1.8<z<8$, we show that the effect of lensing can become significant. Hence, if a population of very-high- $z$ supernovae was ever discovered, it would be crucial to fully understand the effect of lensing, before these SNe could be used to constrain cosmological models. We show that the distance moduli $m-M$ for an open CDM universe and a $\Lambda$ CDM universe are comparable at $z>2$. Therefore if supernovae up to these redshifts were ever discovered, it is still the ones in the range $0.3<z<1$ that would distinguish these two models.
\end{abstract}

Subject headings: cosmology: theory — gravitational lensing — large-scale structure of universe - supernovae

\section{INTRODUCTION}

High-redshift supernovae have become a major tool in modern cosmology. By measuring their apparent magnitudes, we can estimate their luminosity distances $d_{L}$ (see Tonrv et al. 2003; Barris et al. 2004; Riess et al. 2004, and references therein). Since the relationship between $d_{L}$ and the redshift $z$ depends on the cosmological parameters, observations of distant SNe can constrain the cosmological model. Prior to the announcement of the WMAP results (Bennett et al. 2003), observations of high- $z$ SNe provided the most compelling evidence of the existence of a nonzero cosmological constant. Since then, they have been used in combination with the WMAP data to refine the determination of the cosmological parameters.

The luminosity distances $d_{L}$ are determined by combining the observed fluxes $F$ with estimates of the SNe luminosities $L$. Uncertainties in $d_{L}$ are caused by uncertainties in $L$, because $\mathrm{SNe}$ are not perfect standard candles. The flux $F$ is much easier to measure, but for distant sources the value of $F$ might be altered by gravitational lensing caused by the intervening distribution of matter. For instance, a magnification $\mu>1$ would result in a increase in $F$, and an underestimation of $d_{L}$.

Estimating the effect of lensing on the statistics of high- $z$ supernovae is a complex problem. Using either an analytical model or ray-tracing simulations, we can estimate the effect of lensing of a large number of sources in a statistical sense. We would then need to redo the error analysis on the SNe data to include in a

\footnotetext{
${ }^{1}$ Département de physique, de génie physique et d'optique, Université Laval, Québec, Canada, G1K 7P4

${ }^{2}$ Department of Astronomy and Bosscha Observatory, Bandung Institute of Technology, Bandung, Indonesia
} 
consistent way the effect of lensing. This would be a very complex task, and in this paper we have chosen a much simpler approach. Our goal is not to obtain a precise estimate of the error introduced by lensing, but rather to assess the importance of this effect: is it dominant, important, or negligible, and for what range of redshift? and how does it affect the discrimination between different cosmological models? To answer these questions, we take at face value the published results of Type Ia SNe, including their error bars which account for every source of uncertainty but gravitational lensing. Then, we include a posteriori the effect of lensing, to estimate the change in the errors. This approach is not rigorous at all, and does not constitute a substitute for a rigorous treatment of the errors. But it has the great advantage of simplicity. We do not have to redo the detailed error analysis performed by the high-redshift SNe groups, and, more importantly, our conclusions will not be tied to any particular sample or particular data reduction and error analysis technique used by any particular group. We are seeking to make generic statements about the importance of lensing (or lack of) that are relevant to any current or future sample of high- $z$ SNe.

The lensing of distant supernovae has been the focus of several recent studies. In an early study, Wambsganss et al. (1997) used ray-tracing experiments to estimate the effect of weak lensing on the determination of the deceleration parameter $q_{0}$. Ménard \& Dalal (2005), Dodelson \& Vallinotto (2006), and Munshi \& Valageas (2006) focused on SNe as a mean to study the nature of weak lensing. The issue of determining the cosmological parameters for distant $\mathrm{SNe}$, and how this determination is affected by lensing, was addressed by Wang (2005) who used semi-analytical models to determine the magnification distribution function, Holz \& Linder (2005) who used Monte Carlo ray-tracing simulations to study the effect of weak and strong lensing, and Gunnarsson et al. (2006) and Jönsson et al. (2006), who estimated the effect on lensing along individual lines of sight by considering the properties of foreground galaxies in the same direction. These various studies concluded that the effect of lensing on current determinations of the cosmological parameters is small. Aldering et al. (2006) discussed the effect of gravitational lensing on a population of SNe at $z>1.7$.

What distinguishes our approach is mostly its simplicity. Our calculations depend on very few assumptions, and this implies a certain amount of robustness to our results. Even though we rely on numerical simulations, this work should be regarded as a back-of-the-envelope calculation, whose purpose is to obtain a qualitative estimate of the effect of lensing on the determination of cosmological parameters by distant SNe. Using ray-tracing experiments, rather than a semi-analytical approach, enables us to extend our study to redshifts much higher than the ones considered by Wang (2005) and Holz \& Linder (2005).

This paper is organized as follow: In $\S 2$, we describe our calculation of the magnification distribution $P(\mu)$, and how to estimate that distribution at any redshift $z$. In $\S 3$, we describe the real and mock samples of supernovae we use for our calculations. Results are presented in $\S 4$. In $\S 5$, we address various observational issues. Summary and conclusion are presented in $\S 6$.

\section{THE MAGNIFICATION DISTRIBUTION FUNCTION}

\subsection{Simulations}

We have developed a multiple lens-plane algorithm to study light propagation in inhomogeneous universes (Premadi, Martel, \& Matzner 1998; Martel, Premadi, \& Matzner 2000; Premadi et al. 2001a,b). In this algorithm, the space between the observer and the sources is divided into a series of cubic boxes of comoving size $128 \mathrm{Mpc}$, and the matter content of each box is projected onto a plane normal to the line of sight. The trajectories of light rays are then computed by adding successively the deflections caused by each 
plane.

To use this algorithm, we need to provide a description of the matter distribution along the line of sight. Matter is divided into two components: background matter and galaxies. We use a $\mathrm{P}^{3} \mathrm{M}$ algorithm (Hockney \& Eastwood 1981) to simulate the distribution of background matter. The simulations used $64^{3}$ equal-mass particles and a $128^{3} \mathrm{PM}$ grid, inside a comoving volume of size $128 \mathrm{Mpc}$. The matter distribution in the different cubes along the line of sight then corresponds to the state of the simulation at different redshifts 1 We then use a Monte Carlo method for locating galaxies into the computational volume (Martel. Premadi. \& Matzner 1998; Premadi. Martel. \& Matzner 1998). Galaxies are located according to the underlying distribution of background matter. Morphological types are ascribed according to the morphology-density relation (Dressler 1980). Galaxies are modeled as nonsingular isothermal spheres, with rotation velocities and core radii that vary with luminosity and morphological types.

We consider three Cold Dark Matter (CDM) cosmological models: (1) a flat, cosmological constant model $(\Lambda \mathrm{CDM})$ with $\Omega_{0}=0.27, \lambda_{0}=0.73$, and $H_{0}=71 \mathrm{~km} \mathrm{~s}^{-1} \mathrm{Mpc}^{-1}$. This model is in agreement with the results of the WMAP satellite (Bennett et al. 2003). (2) a low-density model with $\Omega_{0}=0.3, \lambda_{0}=0$, and $H_{0}=75 \mathrm{~km} \mathrm{~s}^{-1} \mathrm{Mpc}^{-1}$. (3) a matter-dominated model with $\Omega_{0}=1, \lambda_{0}=0$, and $H_{0}=75 \mathrm{~km} \mathrm{~s}^{-1} \mathrm{Mpc}^{-1}$. For each model, we consider sources at 8 different redshifts: $z_{s} \simeq 1,2,3,4,5,6,7$, and 82 For each combination model-redshift, we performed 10-20 ray tracing experiments. Each experiment consists of propagating a square beam of $101 \times 101=10,201$ rays back in time from the present to redshift $z_{s}$, through the matter distribution. The rays in the beam were widely separated, by 6 arc minutes, and therefore sampled different regions of space. We computed the magnification matrix $\mathbf{A}$ along each ray. The magnification $\mu$ is then given by

$$
\mu=\frac{1}{\operatorname{det} \mathbf{A}} .
$$

Figure 1 shows the distribution of magnifications for the $\Lambda$-model. The distribution peaks at $\mu<1$, and is strongly skewed. The width of the distribution increases with the source redshift. The distributions for the other two models are qualitatively similar.

\subsection{Standard Deviation and Magnification Distribution}

We have determined the distributions $P(\mu)$ at some particular redshifts $z_{s}$. Since SNe do not cooperate by going off only at these redshifts, we now want to interpolate between these distributions, to obtain $P(\mu)$ at any redshift. First, for each model and each source redshift $z_{s}$ we considered, we compute the standard deviation $\sigma_{\mu}$ of the magnification distribution $P(\mu)$. The values are shown in Figure 2 , We use an empirical fit of the form

$$
\sigma_{\mu}=\frac{b z}{1+c z}
$$

where the values of $b$ and $c$ are given in Table 1 . This enables us to estimate the values of $\sigma_{\mu}$ at any redshift. Using the stochastic universe method (SUM) of Holz \& Wald (1998), Holz \& Linder (2005) derived a linear relation between $\sigma_{\text {eff }}$ and $z$ in the range $0 \leq z \leq 2$, for a $\Lambda$ CDM model, where $\sigma_{\text {eff }}$ is the effective

\footnotetext{
${ }^{1}$ In practice, we combine cubes from different simulations in order to avoid periodicities along the line of sight. See also the interesting alternative suggested by Vale \& White (2003).

${ }^{2}$ The exact values of the source redshifts depend on the locations of the lens planes, which vary among models.
} 


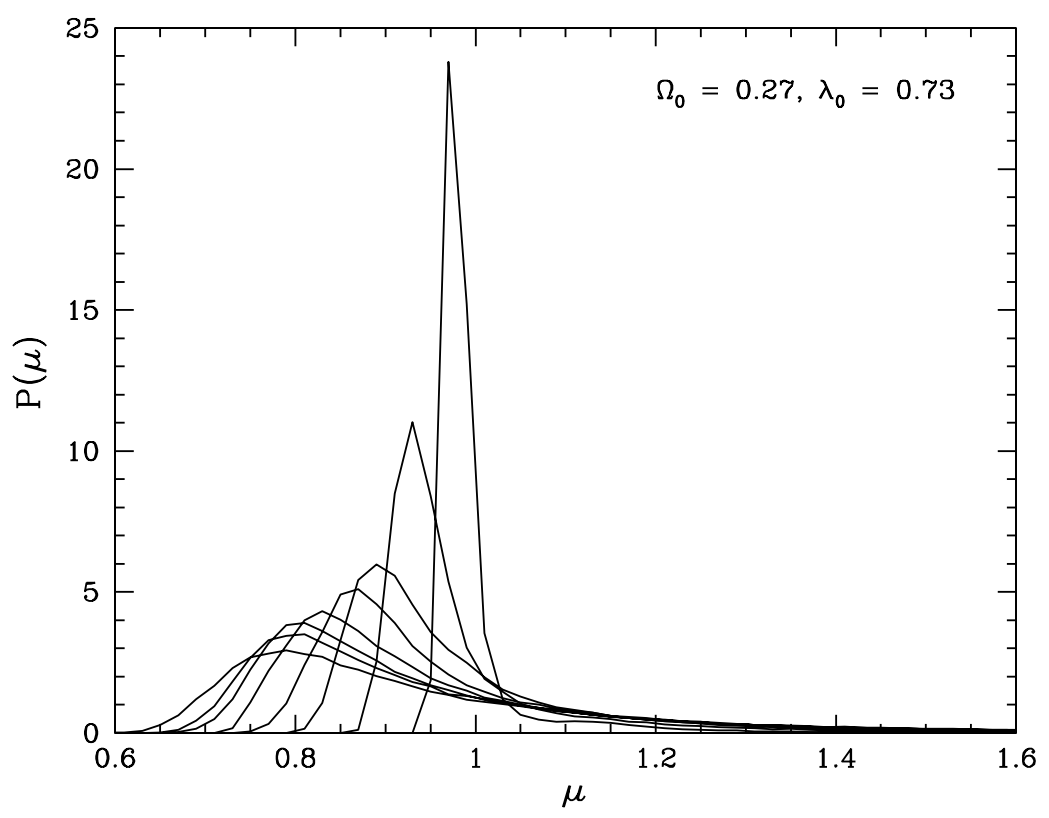

Fig. 1.- Distribution of magnifications for the $\Lambda \mathrm{CDM}$ model. The various curves correspond to different source redshifts: $z_{s}=1$ (narrowest curve), $2,3,4,5,6,7$, and 8 (widest curve).

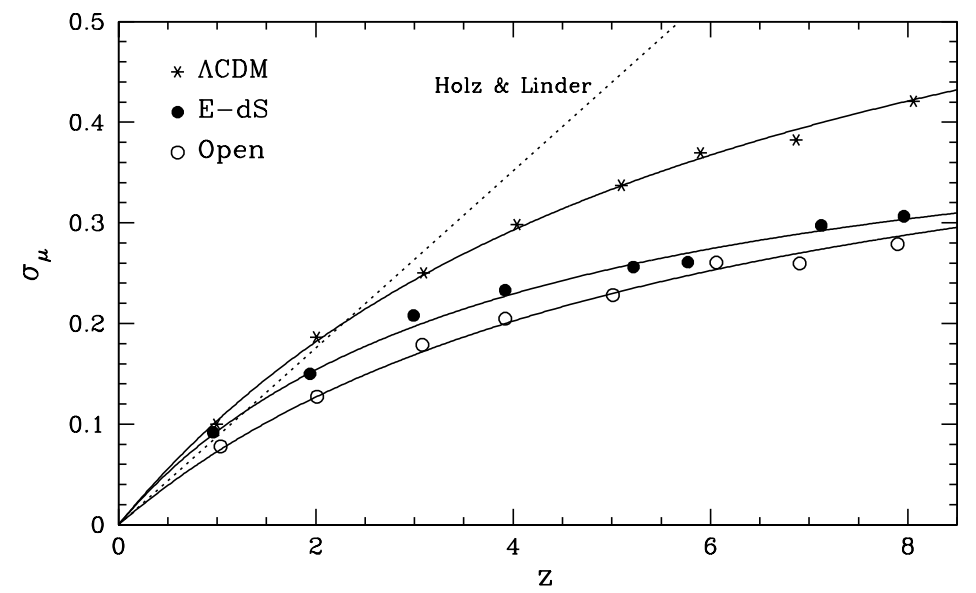

Fig. 2.- Standard deviation $\sigma_{\mu}$ versus redshift, for all three models considered. The solid lines show empirical fits of the form $\sigma_{\mu}=b z /(1+c z)$. The dotted line shows the relation derived by Holz \& Linder (2005).

Table 1. Coefficients of Approximation for $\sigma_{\mu}$

\begin{tabular}{lccccc}
\hline \hline Model & $\Omega_{0}$ & $\lambda_{0}$ & $H_{0}\left[\mathrm{~km} \mathrm{~s}^{-1} \mathrm{Mpc}^{-1}\right]$ & $b$ & $c$ \\
\hline$\Lambda$ CDM & 0.27 & 0.73 & 71 & 0.120 & 0.16 \\
Open & 0.30 & 0.00 & 75 & 0.085 & 0.17 \\
EdS & 1.00 & 0.00 & 75 & 0.117 & 0.26 \\
\hline
\end{tabular}


standard deviation of a single measurement, which is not the same thing as our standard deviation $\sigma_{\mu}$. We plotted their result in Figure 2 for comparison. There is a fairly good agreement between the two methods at redshifts $z \leq 2$. The linear relation has a slope of 0.088 . Our empirical fit for the $\Lambda \mathrm{CDM}$ mode 3 has a slope that varies from 0.120 to 0.069 in the range $z=0-2$.

To determine $P(\mu)$ at any redshift $z$, we interpolate between the distributions we have already determined. Consider two known distributions $P_{1}(\mu)$ and $P_{2}(\mu)$ at redshifts $z_{1}$ and $z_{2}$ that bracket $z$. These distributions satisfy the properties

$$
\begin{aligned}
& \int_{0}^{\infty} P_{i}(\mu) d \mu=1, \\
& \int_{0}^{\infty} \mu P_{i}(\mu) d \mu=1, \\
& \int_{0}^{\infty}(\mu-1)^{2} P_{i}(\mu) d \mu=\left(\sigma_{\mu}^{2}\right)_{i},
\end{aligned}
$$

where $i=1,2$. We define a new distribution,

$$
P(\mu)=\frac{\left[\left(\sigma_{\mu}^{2}\right)_{2}-\sigma_{\mu}^{2}\right] P_{1}(\mu)+\left[\sigma_{\mu}^{2}-\left(\sigma_{\mu}^{2}\right)_{1}\right] P_{2}(\mu)}{\left(\sigma_{\mu}^{2}\right)_{2}-\left(\sigma_{\mu}^{2}\right)_{1}} .
$$

We can easily check that this distribution also satisfies the relations (3)-(15). This enables us to estimate the magnification distribution $P(\mu)$ at any redshift $z$. We first determine $\sigma_{\mu}(z)$ from equation (2), and then substitute it in equation (6) to get $P(\mu)$ at that redshift.

\section{THE SUPERNOVAE CATALOGS}

\subsection{The Tonry et al. Sample}

Observations of high-redshift supernovae provide an estimate of the luminosity distance $d_{L}$. These results are reported in various form in the literature. Some authors express their measurements in terms of effective magnitudes of distance moduli. The High-z Supernova Search Team (Tonry et al. 2003; Barris et al. 2004; Riess et al. 2004) express their measurements in the following form,

$$
\log \left(d_{L} H_{0}\right)=a \pm \delta_{a}
$$

where $H_{0}$ is the Hubble constant, $a$ is the "measurement," and $\delta_{a}$ is the "intrinsic uncertainty," which includes every possible source of error, except gravitational lensing. In this expression, $d_{L} H_{0}$ is expressed in units of kilometers per second. These authors actually use the notation $\left\langle\log \left(d H_{0}\right)\right\rangle$ for $a$ and \pm for $\delta_{a}$.

In this paper, we work with the sample of Tonry et al. (2003). This is not the most up-to-date sample, but it is sufficient for our purpose. This sample is comprised of 230 Type Ia SNe in the redshift range $0<z<1.8$, with 79 of them being located at redshifts $z>0.3$ (including 5 at redshifts $z>0.9$ ). The values of $a$ and $\delta_{a}$ can be read directly in the $8^{\text {th }}$ and $9^{\text {th }}$ columns of their Table 8 , respectively.

\footnotetext{
${ }^{3}$ We use $\Omega_{0}=0.27 ;$ Holz \& Linder 2005$)$ used $\Omega_{0}=0.28$.
} 


\subsection{A Mock Catalog of Very-high $z$ Supernovae}

We generated a mock catalog of $43 \mathrm{SNe}$ in the range $1.8<z<8.1$. For each "supernova," we need to choose a redshift $z$, a measured value $a$, and an uncertainty $\delta_{a}$. There is of course no rigorous method for doing that, since these SNe do not exist. To provide a good coverage of the redshift range of interest, we used 43 equally-spaced values of $z$ between $z=1.8$ and 8.1. To determine $\delta_{a}$, we first plotted $\delta_{a}$ versus $z$, for the Tonry et al. (2003) sample, to look for trends. There is a large number of SNe with $z<0.1, \delta_{a}<0.05$. If we focus on the $79 \mathrm{SNe}$ at redshift $z>0,3$, we do not see any obvious trend, and in particular $\delta_{a}$ does not appear to increase with redshift. So we chose, somewhat arbitrarily, the $9 \mathrm{SN} 4$ at $z>0.828$. For these SNe, the mean and standard deviation of the uncertainties are $\bar{\delta}_{a}=0.0631$ and $\sigma_{\delta}=0.0113$, respectively. We then chose the values of $\delta_{a}$ for our mock SNe randomly, by drawing them from a normal distribution with mean $\bar{\delta}_{a}$ and standard deviation $\sigma_{\delta}$. This ensures a smooth transition between the real and mock samples.

To determine $a$, we assume that the underlying cosmology corresponds to a $\Lambda$ CDM universe (as supported by the real sample). We then use

$$
a=a_{\Lambda}+\Delta a,
$$

where $a_{\Lambda} \equiv \log \left(d_{\Lambda} H_{0}\right)$ is the actual value of $\log \left(d_{L} H_{0}\right)$ in a $\Lambda \mathrm{CDM}$ universe, and $\Delta a$ is a random number drawn from a normal distribution with mean 0 and standard deviation given by the value of $\delta_{a}$ we just calculated.

\section{THE EFFECT OF LENSING ON STATISTICS OF HIGH-Z SUPERNOVAE}

\subsection{Compounding the Errors}

As we explained in $\S 1$, our goal is not to perform a rigorous error analysis of the uncertainties resulting from the possibility of lensing, but rather to estimate a posteriori the effect of lensing on the uncertainties already present in the analysis.

We estimate the effect of lensing as follows: the distances of high- $z$ supernovae are reported in the literature in the format given by equation (7), where $\delta_{a}$ is the intrinsic uncertainty (i.e. not caused by lensing). The distance $d_{L}$ is related to the luminosity $L$ and flux $F$ by

$$
F=\frac{L}{4 \pi d_{L}^{2}} .
$$

We use equations (7) and (9) to eliminate $d_{L}$, and get

$$
L^{1 / 2} H_{0}=10^{a} 10^{ \pm \delta_{a}}(4 \pi F)^{1 / 2} .
$$

The effect of lensing will be to modify the flux $F$. To account for it, we replace $F$ by $F \pm \Delta F$ in equation (10), and perform a Taylor expansion to first order in $\Delta F$,

$$
L^{1 / 2} H_{0}=10^{a} 10^{ \pm \delta_{a}}(4 \pi F)^{1 / 2}\left(1 \pm \frac{\Delta F}{2 F}\right) .
$$

\footnotetext{
${ }^{4}$ We deliberately avoided sn97G and sn76cl, whose uncertainties are much larger than those of any SNe at comparable redshift.
} 
This expression reduces to

$$
\log \left(d_{L} H_{0}\right)=a \pm \delta_{a} \pm \frac{\Delta F}{2 F \ln 10} .
$$

The last term represents the uncertainty due to lensing. For a given supernova with magnification $\mu$, $\Delta F / F=\mu-1$. Of course, we will never know the value of $\mu$ for a single source. But for a large number of sources, we can use statistics. First, the simplest, lowest-order approximation for a "typical" value of $\mu$ is $\mu=\langle\mu\rangle \pm \sigma_{\mu}=1 \pm \sigma_{\mu}$, or equivalently $\Delta F / F \approx \sigma_{\mu}$. Equation (12) reduces to

$$
\log \left(d_{L} H_{0}\right)=a \pm \delta_{a} \pm \delta_{\mu}
$$

where $\delta_{\mu}(z)=\sigma_{\mu}(z) / 2 \ln 10$ can be computed using the empirical relations plotted in Figure 2, We use the values of $a$ and $\delta_{a}$ reported by Tonry et al. (2003) (their Table 8). In Figure 3 , we plot the ratio $\delta_{\mu} / \delta_{a}$ versus $z$ (left of the dotted lines). This quantity increases with redshift, but never gets higher than 0.5 for the Tonry et al. sample. Furthermore, we shall assume that $\delta_{a}$ and $\delta_{\mu}$ are statistically independent, and combine them in quadrature, using

$$
\delta=\left(\delta_{a}^{2}+\delta_{\mu}^{2}\right)^{1 / 2},
$$

where $\delta$ is the total error. The contribution of lensing to this error is then of order $25 \%$ at most.

For each supernova, we compute the quantity $\Delta(m-M)$ (deviation of the difference between apparent and absolute magnitude, relative to an empty universe), using

$$
\Delta(m-M)=5 \log \left(d_{L} H_{0}\right)-5 \log \left(d_{L} H_{0}\right)_{\text {empty }}=5 \log \left(d_{L} H_{0}\right)-5 \log \left[c z\left(1+\frac{z}{2}\right)\right] .
$$

We then average the quantities $\Delta(m-M)$ and $\delta$ in redshift bins, using

$$
\begin{aligned}
{[\Delta(m-M)]_{j} } & =\Sigma_{i} w_{i} \Delta(m-M) / \Sigma_{i} w_{i} \\
\delta_{j} & =\left(1 / \Sigma_{i} w_{i}\right)^{1 / 2}
\end{aligned}
$$

where

$$
w_{i}=1 / \delta_{i}^{2}
$$

and the sums are over all data points $i$ in bin $j$ (note: eq. [17 comes from $1 / \delta_{j}^{2}=\sum_{i}\left[1 / \delta_{i}^{2}\right]$ ). Notice that this method of averaging is much fancier than what appears to be done in the supernovae papers. For instance, Figure 9 of Tonry et al. (2003) shows an averaging over redshift bins which is based on the median of the data and apparently does not take into account the uncertainties $\delta_{a}$ on the individual supernovae.

Figure 4 shows a Hubble diagram [deviation $\Delta(m-M)$ versus redshift]. The data points and error bars on the left hand side of the dotted lines correspond to the values given by equations (16) and (17), respectively. The three curves, from top to bottom, show the exact results for the $\Lambda$ CDM, low-density, and matter-dominated models, respectively. The results support the flat $\Lambda \mathrm{CDM}$ model and exclude the other models considered. The other panels of Figure 4 show the effect of lensing (the three models have to be plotted separately, because the correction due to lensing, which uses the relations plotted in Fig. 2, is 

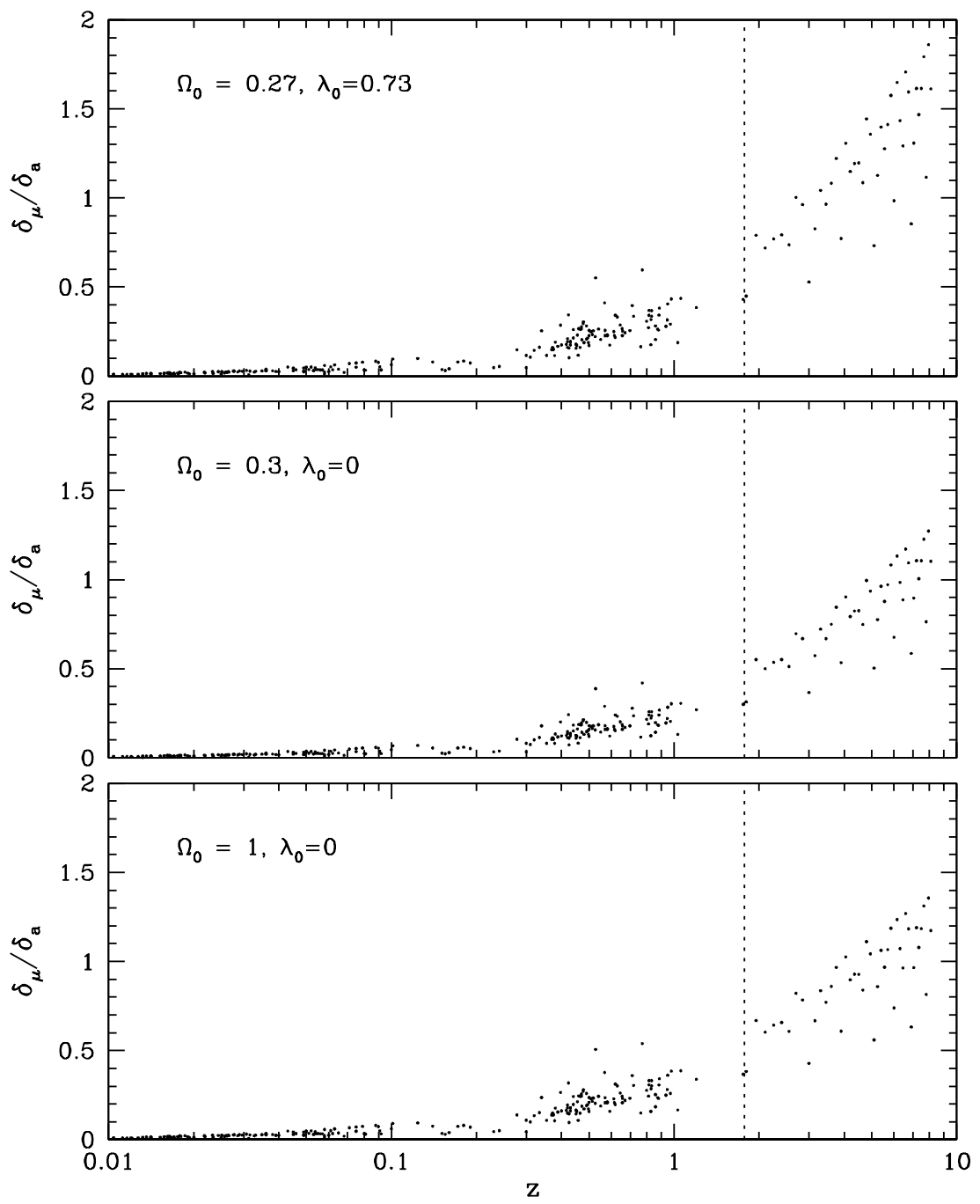

Fig. 3.- Ratio $\delta_{\mu} / \delta_{a}$ versus redshift. The dotted lines separate the real data of Tonry et al. (left side) from the mock, high-redshift data (right side). 

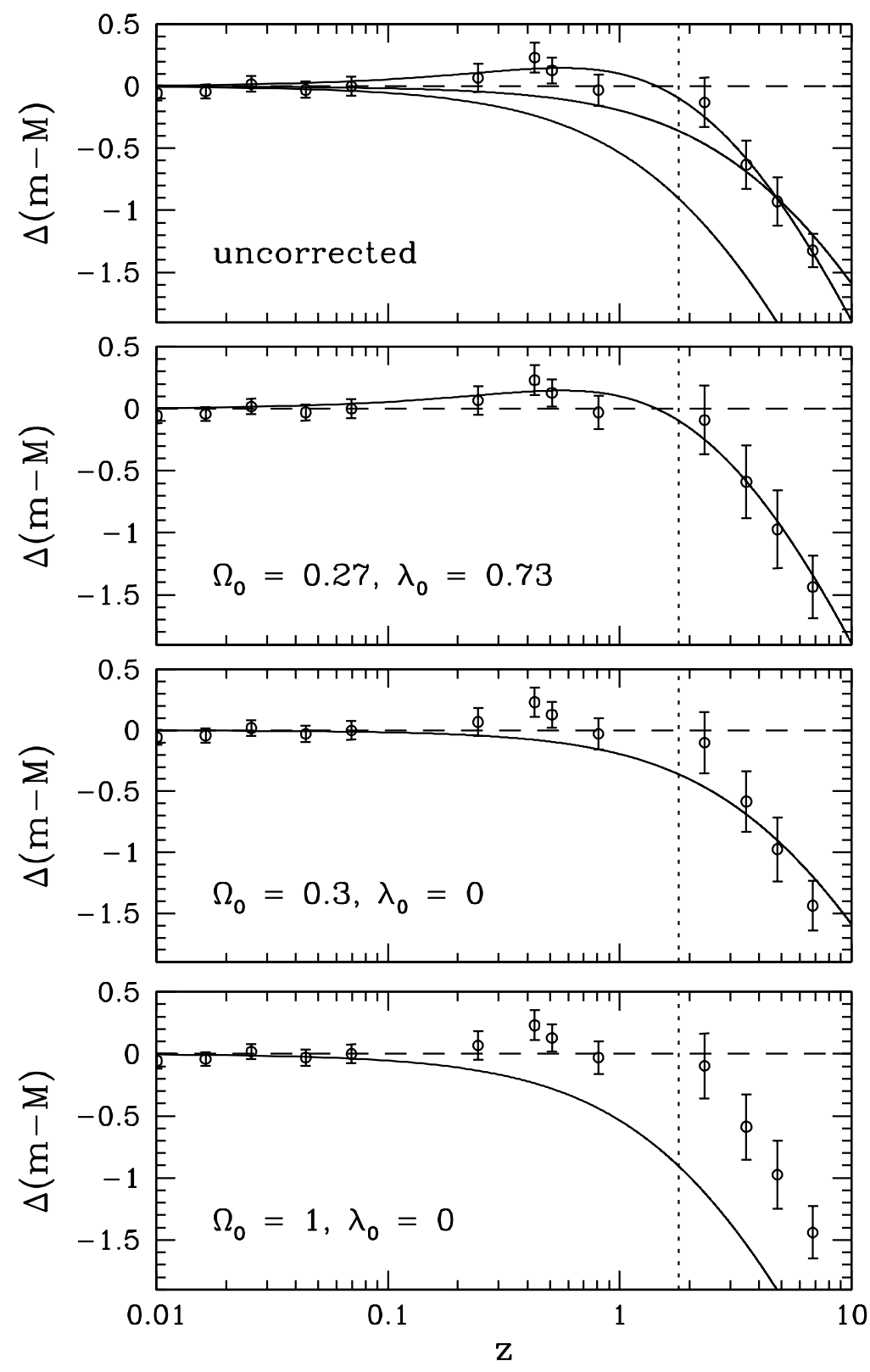

Fig. 4.- Hubble diagram showing the magnitude deviation $\Delta(m-M)$ relative to an empty universe, for the three models considered. The dotted lines separate the Tonry et al. (2003) data (on the left) from the mock data (on the right). In the top panel, the three curves, from top to bottom, show the analytical result for the cosmological models $\left(\Omega_{0}, \lambda_{0}\right)=(0.27,0.73),(0.3,0.0)$, and $(1.0,0.0)$, respectively. The error bars show $90 \%$ confidence level. The last three panels reproduce the data of the top panel, but have been corrected to account for lensing. Since this correction is model-dependent, the three models are plotted on separate panels. 
model-dependent). This effect is totally negligible. The largest correction to the error bars is about $10 \%$ for the highest redshift bin, for the $\Lambda$ CDM model.

Clearly, the potential error introduced by lensing is negligible in comparison to the intrinsic error in the measurement, at least for SNe at redshifts $z<1$.8. Gunnarsson et al. (2006) and Jönsson et al. (2006) reach the same conclusion, We now estimate the effect of lensing on a yet-undiscovered population of very-high- $z$ SNe, using our mock catalog. The ratios $\delta_{\mu} / \delta_{a}$ are plotted in Figure 3, on the right hand side of the dotted lines. The effect of lensing rapidly becomes important at redshift $z>2$, especially for the $\Lambda$ CDM models. We find many SNe with $\left|\delta_{\mu}\right|>\delta_{a}$, that is, the correction due to lensing is larger than the intrinsic uncertainty.

The points located on the right hand side of the dotted lines in Figure 4 shows the results for the mock data. The error bars get significantly bigger when lensing is included. Furthermore, at redshift $z \approx 3$, it becomes very difficult to distinguish the open, low density model from the cosmological constant model, because the theoretical curves intersect. Keeping in mind the caveat that the mock catalog was built under the assumption that the underlying cosmology was $\Lambda \mathrm{CDM}$, we see that the Einstein-de Sitter model is totally ruled out by $\mathrm{SNe}$ at $z>2$, but the open model is not. Indeed, it is clear that $\mathrm{SNe}$ at $z>2$ would be rather useless in distinguishing an open CDM and a $\Lambda \mathrm{CDM}$ model: the theoretical curves get closer, whereas the error bars become larger. It is, interestingly, the SNe in the redshift range $0.3<z<1$ that would still provide the best discriminant between these two models, and data in that redshift range are already available.

\subsection{Monte Carlo Approach}

The calculation presented in the previous section relies entirely in the standard deviation $\sigma_{\mu}$ for estimating the uncertainties caused by lensing. This approach would probably be sufficient if the distributions of magnifications $P(\mu)$ were gaussian. However, for sources at large redshifts, $P(\mu)$ is strongly skewed, as Figure 1 shows. The large majority of sources are demagnified, as the light reaching the observer travels mostly through underdense regions of the universe, while a few sources are strongly magnified, especially those which happen to be aligned with a massive galaxy at intermediate redshift.

To account for the distribution of magnifications, we consider all SNe at redshifts $z>0.9$ (5 from the Tonry sample, 43 from the mock catalog). For each one, we determine the distribution $P(\mu)$ at its redshift $z$, using equation (6), and then choose a magnification $\mu$ by drawing it randomly from the distribution $P(\mu)$. We then compute $\delta_{\mu}=\Delta F / 2 F \ln 10=(\mu-1) / 2 \ln 10$. The resulting ratios $\left|\delta_{\mu}\right| / \delta_{a}$ are plotted in Figure 5 , Comparing with Figure 3, we find only a few SNe for which this ratio exceeds unity. For all redshifts and all models, we find that the distributions $P(\mu)$ peak at a value $\mu_{\text {peak }}<1$ such that $\left|\mu_{\text {peak }}-1\right|<\sigma_{\mu}$. Hence, setting $\Delta F / F=\sigma_{\mu}$ (instead of $\Delta F / F=\mu-1$ ), as we did in $\S 4.1$, overestimates the effect of lensing for most SNe. However, the distributions are very skewed, and as a result a few SNe are highly magnified, as Figure 5 shows.

Figure [6 shows the resulting Hubble diagram. Comparing with Figure 4, we find that the error bars are significantly smaller. The effect of lensing is less important when we use the actual distribution of magnifications $P(\mu)$, and not only its standard deviation. However, the results at $z>1.8$, which assume an underlying $\Lambda \mathrm{CDM}$ cosmology, still cannot rule out the open CDM model; the error bars are still too large. 

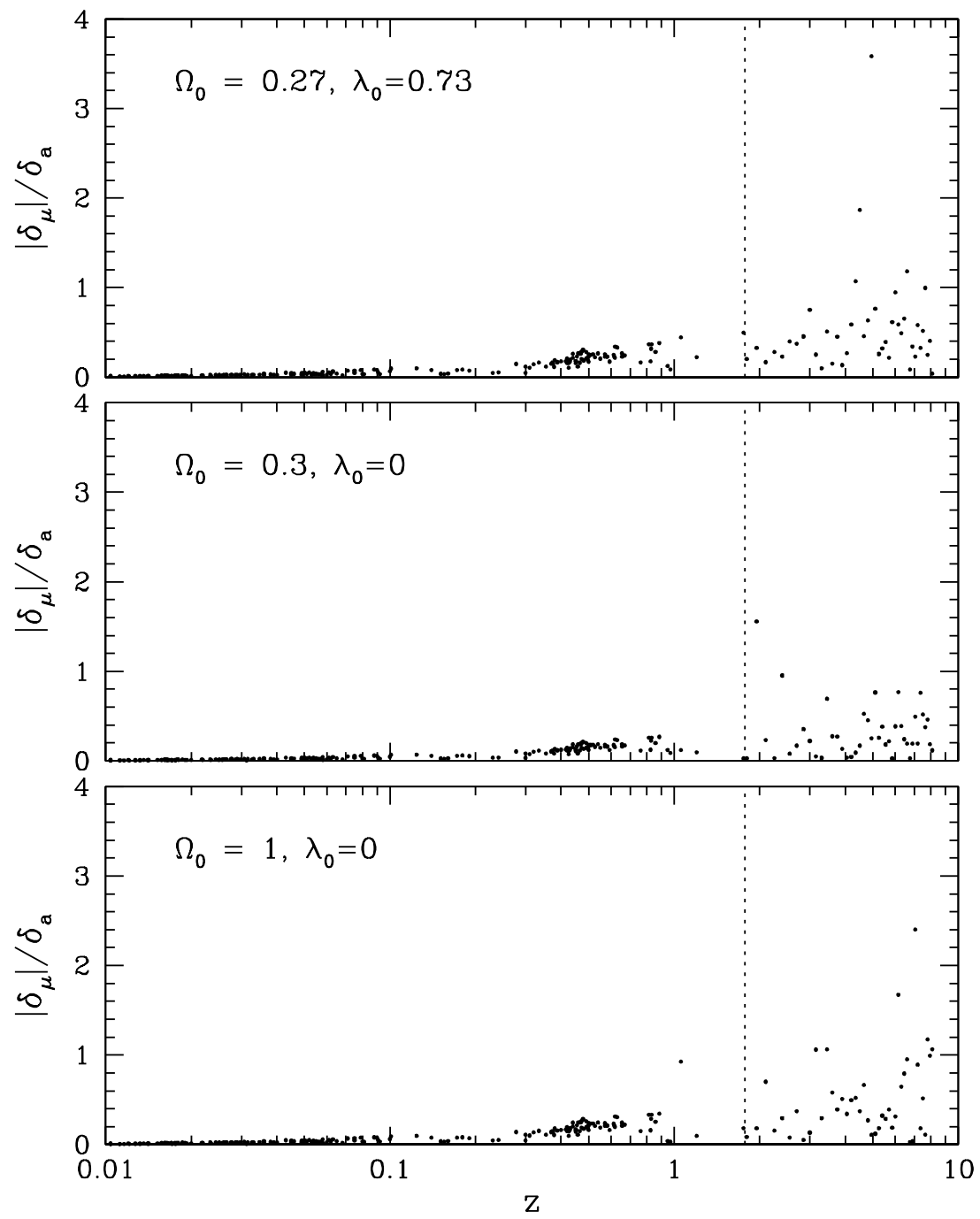

Fig. 5.- Same as Figure 3, but with $\delta_{\mu}$ computed using the Monte Carlo approach for all SNe (real or mocked) at redshift $z>0.9$. 

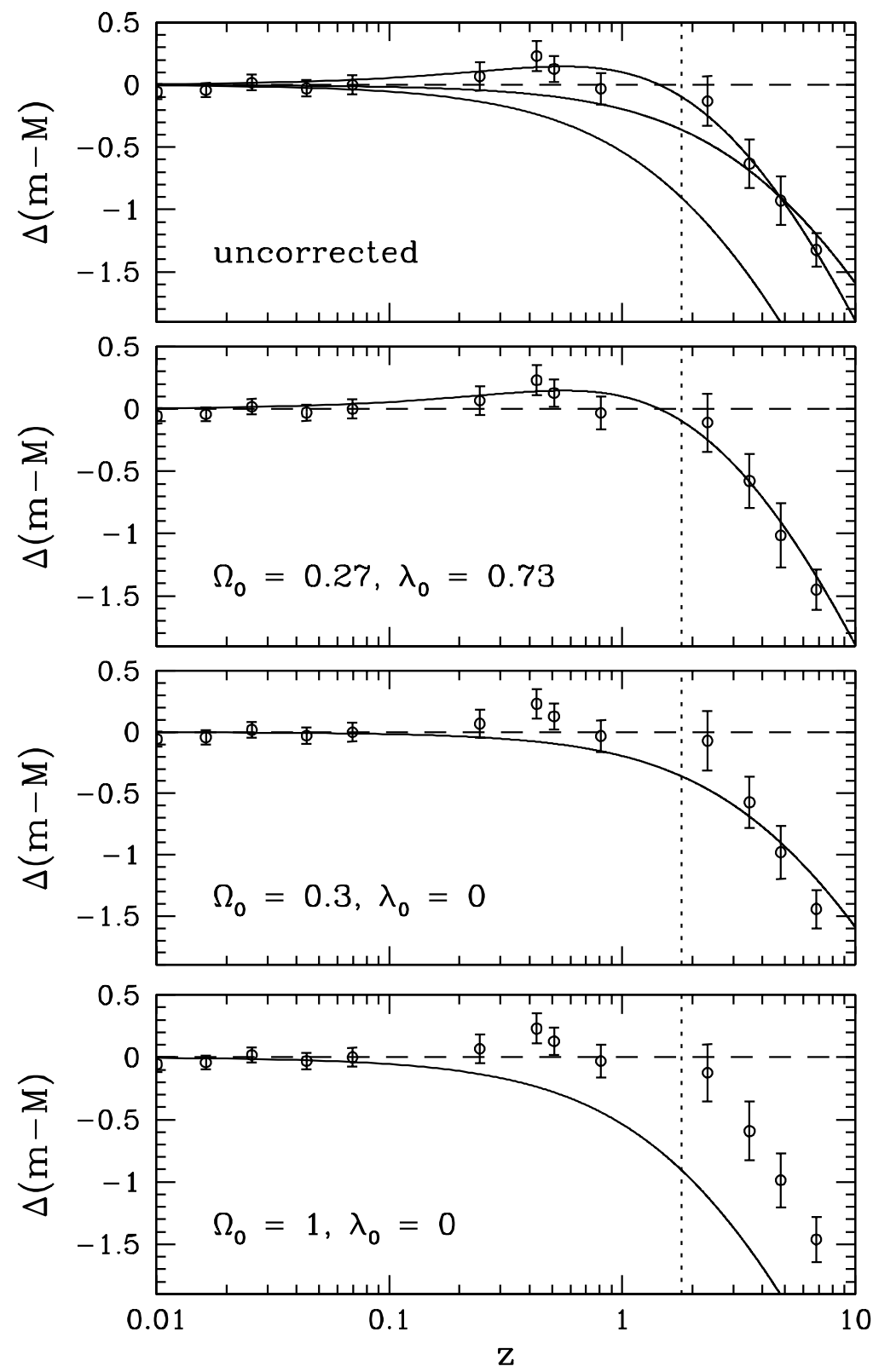

Fig. 6.- Same as Figure 4, with errors bars computed using the Monte Carlo approach. 


\section{DETECTION LIMIT AND BIASING}

So far, we have assumed that any supernova, with any value of $z$ and $\mu$, can be observed. This assumption is probably valid over the range $0<z<2$, which includes all the current observations. But as the redshift gets higher, it becomes increasingly difficult to observe supernovae with current and even future telescopes, because of the combined effect of the flux reduction and the light being shifted to the near infrared. It might just be impossible to detect a supernova at redshift $z>2$ unless, of course, it is magnified by lensing. Marri \& Ferrara (1998) argue that, with magnification taken into account, it might be possible to observe Type II SNe at redshifts up to $z=10$. Of course, if only the highly-magnified SNe are observable, this introduces biasing, an effect that we must take into account.

Here, we do not want to perform a detailed analysis similar to the one of Marri \& Ferrara (1998), but just to get a rough estimate of the importance of biasing. The key results of the analysis of Marri \& Ferrara (1998) are shown in their Figure 7, which shows, as a function of redshift, the AB apparent magnitude in the J, K, L, and M bands, with and without lensing. They also plot the expected flux limit of JWST5. From this figure, we see that without lensing, the apparent magnitudes are below the detection limit at high redshift. When lensing is included, the magnitudes in the $\mathrm{J}$ and $\mathrm{K}$ bands are above the detection limit. Their analysis was for Type II SNe. Type Ia SNe are typically 1.5 magnitudes brighter, and therefore much easier to detect. Neglecting the details of the spectra, we can simply take Figure 7 of Marri \& Ferrara (1998) and shift all the curves upward by 1.5 magnitudes. We find that, without lensing, Type Ia SNe would be visible in the $\mathrm{J}$ and $\mathrm{K}$ band, and almost visible in the $\mathrm{L}$ band, at redshifts up to $z \sim 8$. With lensing, most SNe would be demagnified, but the reduction in flux is typically of the order $10-20 \%$, that is, a correction of $0.103-0.198$ magnitudes. Hence, all high- $z$ Type Ia SNe should be detectable, using the proper telescope, and therefore we were justified to ignore any biasing effect. This being said, the identification of Type Ia $\mathrm{SNe}$ requires that we obtain a spectrum, and this could be quite challenging at these extreme redshifts.

We have assumed that the typical intrinsic uncertainties $\delta_{a}$ do not grow with redshift for $z>1.8$, based on the absence of obvious trend at $z \lesssim 1$. . If the uncertainties do grow with redshift, our conclusion that understanding the effect of lensing at high redshift would be weakened, in the sense that these data would be rather useless no matter how well lensing is understood. Furthermore, it would reinforce our conclusion that $\mathrm{SNe}$ at intermediate redshifts are more useful to discriminate between different cosmological models.

We should also ask whether Type Ia SNe at redshift $z=8$ can actually exist. For a $\Lambda$ CDM model with a Hubble constant of $71 \mathrm{~km} \mathrm{~s}^{-1} \mathrm{Mpc}^{-1}$, this redshift corresponds to an age of the universe of $650 \mathrm{Myr}$. Subtracting the formation and evolutionary time of the progenitor, we are getting embarrassingly close to the big bang. Type II SNe would be a far better candidate for high- $z \mathrm{SNe}$, since the evolutionary time of their progenitors are much shorter. But then only the few that are magnified would be detectable, and their number might be too small to perform any meaningful statistics on them.

\section{SUMMARY AND CONCLUSION}

We have performed a series of ray-tracing experiments using a multiple lens-plane algorithm. We have determined the distributions of magnifications $P(\mu)$ for sources in the redshift range $0<z<8$, for three different cosmological models. We have used these distributions to estimate the effect of gravitational

\footnotetext{
${ }^{5}$ They called it NGST back then.
} 
lensing on the determination of the cosmological parameters with high-redshift Type Ia supernovae. We used a generic, a posteriori approach which is not tied to any particular sample.

We found that errors introduced by lensing are unimportant for SNe with redshift $z<1.8$. These errors are negligible compared to the intrinsic errors already present in the supernovae data. Since those intrinsic errors do not prevent us from determining the cosmological parameters, the additional errors introduced by lensing have no consequences. A similar conclusion was reached by Aldering et al. (2006).

Using a mock catalog of high- $z \mathrm{SNe}$, extending to $z=8.1$, we showed that the effect of lensing on a hypothetical population of SNe at redshifts $z>2$ could be quite significant, and must be understood before such SNe could be used to constrain cosmological models. Furthermore, the open CDM and $\Lambda$ CDM are difficult to distinguish at that redshift. We showed that, even if SNe at redshift $z \sim 8$ were ever discovered, it is the $\mathrm{SNe}$ in the range $z=0.3-1$ that would still provide the best discriminant between these two models. The data at that redshift already exist, and they support the $\Lambda$ CDM model.

This work benefited from stimulating discussions with Gilbert Holder, Daniel Holz, Eric Linder, Massimo Meneghetti, and Christopher Vale. The calculations were performed at the Texas Advanced Computing Center, University of Texas, and the Laboratoire d'Astrophysique Numérique, Université Laval. HM thanks the Canada Research Chair program and NSERC for support. PP thanks Uroš Seljak for various advice and hospitality during the fruitful visit at the Abdus Salam ICTP in Trieste. 


\section{REFERENCES}

Aldering, G., Kim, A. G., Kowalski, M., Linder, E. V., \& Perlmutter, S. 2006, Astropart. Phys., 27, 213

Barris, B. J. et al. 2004, ApJ, 602, 571

Bennett, C. L. et al. 2003, ApJS, 148, 1

Dodelson, S., \& Vallinotto, A. 2006, PRD, 74, 063515

Dressler, A. 1980, ApJ, 236, 351

Gunnarsson, C., Dahlén, T., Goobar, C., Jönsson, J, \& Mörtsell, E. 2006, ApJ, 640, 417

Hockney, R. W., \& Eastwood, J. W. 1981, Computer Simulation using Particles (New York: McGraw Hill).

Holz, D. E., \& Linder, E. V. 2005, ApJ, 631, 678

Holz, D. E., \& Wald, R. M. 1998, Phy. Rev. D., 58, 063501

Jönsson, J., Dahlén, T., Goobar, A., Gunnarsson, C., Mörtsell, E., \& Lee, K. 2006, ApJ, 639, 991

Marri, S., \& Ferrara, A. 1998; ApJ, 509, 43

Martel, H., Premadi, P., \& Matzner, R. 1998, ApJ, 497, 512

Martel, H., Premadi, P., \& Matzner, R. 2000, ApJ, 537, 28

Ménard, B., \& Dalal, N. 2005, MNRAS, 358, 101

Munshi, D., \& Valageas, P. 2006, preprint (astro-ph/0601683)

Premadi, P., Martel, H., \& Matzner, R. 1998, ApJ, 493, 10

Premadi, P., Martel, H., Matzner, R., \& Futamase, T. 2001a, ApJS, 135, 7

Premadi, P., Martel, H., Matzner, R., \& Futamase, T. 2001b, Pub.A.S.Aus., 18, 201

Riess, A. G. et al. 2004, ApJ, 607, 665

Tonry, J. L. et al. 2003, ApJ, 594, 1

Vale, C., \& White, M. 2003, ApJ, 592, 699

Wambsganss, J., Cen, R., Xu, G., \& Ostriker, J. P. 1997, ApJ, 475, L81

Wang, Y. 2005, JCAP, 503, 5 